Rothe, Ralph; Lemke, Klaus; Plagwitz, Kai-Uwe:

Meßtechnische Probleme bei einem neuen Verfahren zur berührungslosen Augeninnendruckmessung

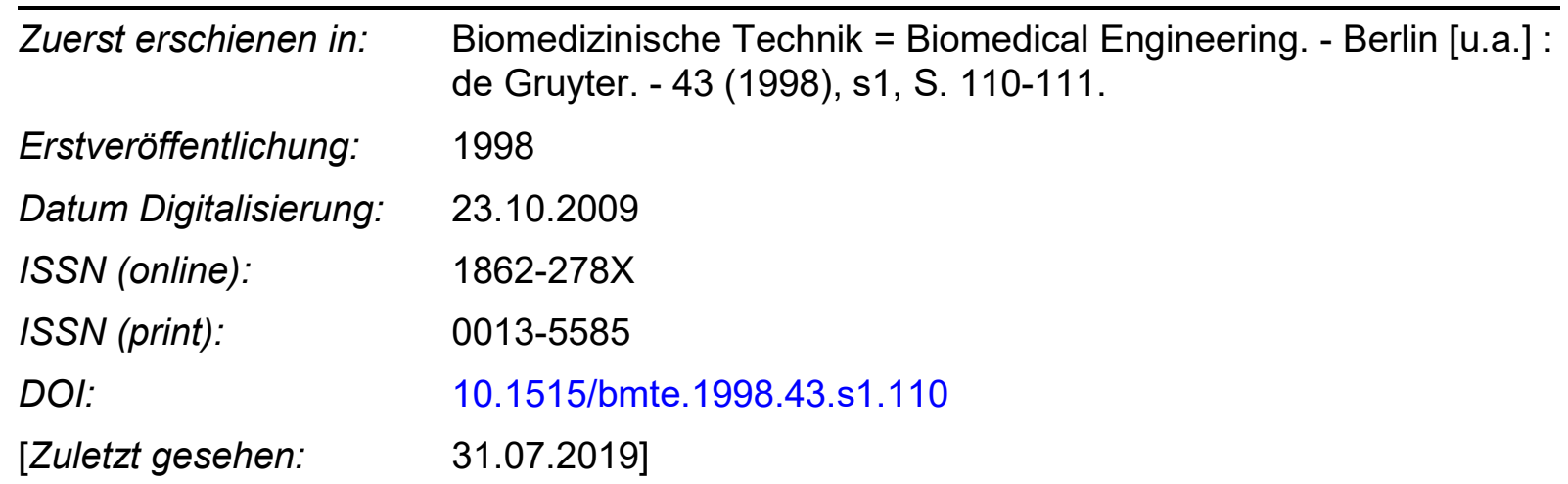

„Im Rahmen der hochschulweiten Open-Access-Strategie für die Zweitveröffentlichung identifiziert durch die Universitätsbibliothek IImenau."

"Within the academic Open Access Strategy identified for deposition by IImenau University Library."

„Dieser Beitrag ist mit Zustimmung des Rechteinhabers aufgrund einer (DFGgeförderten) Allianz- bzw. Nationallizenz frei zugänglich."

"This publication is with permission of the rights owner freely accessible due to an Alliance licence and a national licence (funded by the DFG, German Research Foundation) respectively."

\section{DFG}

Nationallizenzen 


\title{
Meßtechnische Probleme bei einem neuen Verfahren zur berührungslosen Augeninnendruckmessung
}

\author{
Rothe R., Lemke K., Plagwitz K.-U.
}

Technische Universität Ilmenau, Institut für Biomedizinische Technik und Informatik, PF 100 565, D-98684 Ilmenau

\section{EINLEITUNG}

In den Industrienationen ist neben dem Diabetes melitus das Glaukom die häufigste Ursache für eine Erblindung im fortgeschrittenen Alter. Nach wie vor besitzt die Tonometrie zur Diagnostik und Therapiekontrolle der Glaukomerkrankung herausragende Bedeutung, da durch eine frühzeitige Erkennung auffalliger Augeninnendruckwerte wirksame Maßnahmen zur Verhinderung bzw. Begrenzung von Schäden am Sehnerv eingeleitet werden können.

Der Trend geht hin zu Non-ContactTonometrieverfahren, weil Anästhesierung der Kornea und Anfärbung der Tränenflüssigkeit dabei entfallen können. Gegenüber den berührend arbeitenden Geräten haben die Non-Contact-Tonometer eine erhöhte Meßunsicherheit, die aus Positionierungsfehlern, Druckschwankungen aufgrund des Blutpulses und interindividuell unterschiedlichen mechanischen Eigenschaften der Kornea (Korneadicke, -steifigkeit) resultieren.

Mit dem von uns entwickelten Verfahren der NonContact-Tonometrie sollen die Einflüsse der geometrischen und elastischen Eigenschaften der Kornea abgeschätzt und daraus ein Korrekturfaktor für das Tonometriemeßergebnis abgeleitet werden.

\section{MESSAPPARATUR}

Der Grundgedanke des Meßverfahrens ist die Aufnahme des zeitlichen Verlaufs der Verformung der Augenhornhaut bei Einwirken einer definierten Kraft. Dabei soll insbesondere das weitgehend von äußeren Einflüssen freie Zurückschwingen in die Ausgangslage Aussagen über die biomechanischen Eigenschaften der Kornea liefern.

Zur reproduzierbaren Verformung der Kornea dient ein

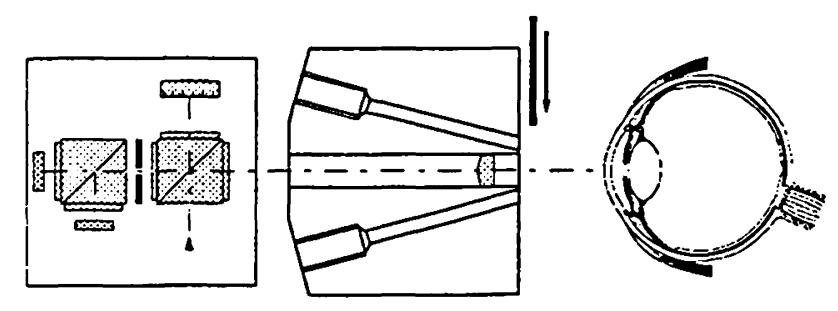

Luftimpulsgenerator in Verbindung mit einem Düsenkopf. Als Alternative dazu wird die Möglichkeit der Verwendung von Luftultraschall zur Deformation der Hornhaut untersucht, wobei bisher allerdings die vom Sender abgegebene Leistung zu gering war. so daß nur unzureichende Wirkungen erzielt werden konnten. Die berührungslose, hochgenaue Messung der Reaktion der Kornea auf die Krafteinwirkung erfolgt mit einem polarisationsoptischen Laserinterferometer. Dieses Interferometer akzeptiert in Grenzen Meßflächenverkippungen und toleriert Lufturbulenzen im Meßarm. Die Funktionsweise des Laserinterferometers macht es erforderlich, daß der Hornhautpol in Richtung der Flächennormalen mit dem Meßstrahl angetastet wird. Aus diesem Grund ist der Düsenkopf als Doppeldüsenanordnung mit zentraler Bohrung für den Laserstrahl ausgeführt. Die Synchronisation der Geräte sowie die Datenübernahme und Darstellung der Verformungskurven erfolgt mit einem Computer.

\section{FUNKTIONSWEISE UND MESSTECHNISCHE PROBLEME}

Der zur Deformation verwendete Druckluftimpuls hat die Form einer Rampe. Nach einem linearen Druckanstieg einstellbarer Länge erfolgt ein sehr steiler Druckabfall. Mit dem Laserinterferometer wird während des gesamten Meßvorganges die Auslenkung des Korneapoles, d.h. sowohl die Verformung während des Staudruckanstieges als auch das Rückschwingen in die Ausgangslage, erfaßt. Die ermittelten Verformungskurven werden hinsichtlich des Anstieges und der Frequenz- und Dämpfungscharakteristik beim Ausschwingen ausgewertet.

Der aufgeprägte Luftimpuls ist von Druckschwingungen infolge von Turbulenzen überlager. die sich in den aufgenommenen Verformungskurien widerspiegeln. Durch die Messung des gesamten zeitlichen Verlaufes können diese jedoch mit Hilfe von Signalanalysefunktionen ausgeglichen werden.

Die schnelle Abschaltung des Luftimpulses wird mit einer Schwenkblende realisiert, da Ventile mit Stellzeiten unter $1 \mathrm{~ms}$ nicht verfugbar sind. Dabei bleibt beim Abschaltvorgang die zentrale Laserstrahldurchführung zur fortlaufenden Messung frei.

Das verwendete Interferometer muß für die Anwendung am Auge bei diesem Tonometrieverfahren 
spezielle Anforderungen erfullen. Zur Vermeidung von Schäden an der Netzhaut darf ein Strahlungsleistungsgrenzwert von $300 \mu \mathrm{W}$ nicht überschritten werden. Um Messungen an der gekrümmten, gering reflektierenden (2,5\%) Kornea zu ermöglichen, muß der Meßstrahl fokussiert werden. Bei den geringen von der Kornea reflektierten Strahlungleistungen, die zur Erzeugung auswertbarer Interferenzmuster bereitstehen, läßt das Interferometer Meßflächengeschwindigkeiten von bis zu $35 \mathrm{~cm} / \mathrm{s}$ ohne Funktionsausfall zu.

Das polarisationsoptische Laserinterferometer bietet zusätzlich die Möglichkeit der ungleichmäßigen Verteilung der Strahlungsleistung auf die Teilarme, so daß der Kontrast des Interferenzbildes optimiert werden kann.

Zu Experimentier- und Prüfzwecken wurde ein mechanisches Augenphantom mit einspannbaren Kalotten, die zur Nachbildung der Kornea dienen, entwickelt [3]. Infolge von Inhomogenitäten der Testkalotten kann es durch die Verformung mit Hilfe der Doppeldüsenanordnung zu Verkippungen der Meßfläche bei Einwirken des Luftimpulses kommen, die zum Ausfall der interferometrischen Messung führen. Durch eine optimierte Gestaltung des Düsenkopfes kann dieser Effekt jedoch in Zukunft minimiert werden.

\section{ERGEBNISSE UND DISKUSSION}

Das neue Meßverfahren wurde im Laboraufbau an Eichkalotten für Non-Contact-Tonometer, am mechanischen Augenmodell und an Schweineaugen mit Erfolg getestet. Dabei ergab sich bei zunehmendem Augeninnendruck immer eine Verringerung des Anstiegs der Verformungskurve und unterschiedliches Frequenz- und Dämpfungsverhalten beim Ausschwingvorgang.

Am mechanischen Augenphantom zeigt sich bei reproduzierbarer Luftimpulscharakteristik ein eindeutiger Zusammenhang zwischen dem eingestellten Augeninnendruck und dem Anstieg der Verformungskurve. Im Ausschwingverhalten weisen Kalotten höherer Wandstärke eine deutlich größere Eigenfrequenz und eine geringfügig höhere Dämpfung auf. Aus dem Anstieg erhält man also Informationen über den Augeninnendruck, und das Ausschwingverhalten liefert Ansätze für die Ableitung einer individuellen Korrekturgröße.

Bei der Kenntnis des zeitlichen Verlaufs des Bewegungsvorganges ist somit eine Augeninnendruckbestimmung auch ohne Applanationsdetektion móglich.

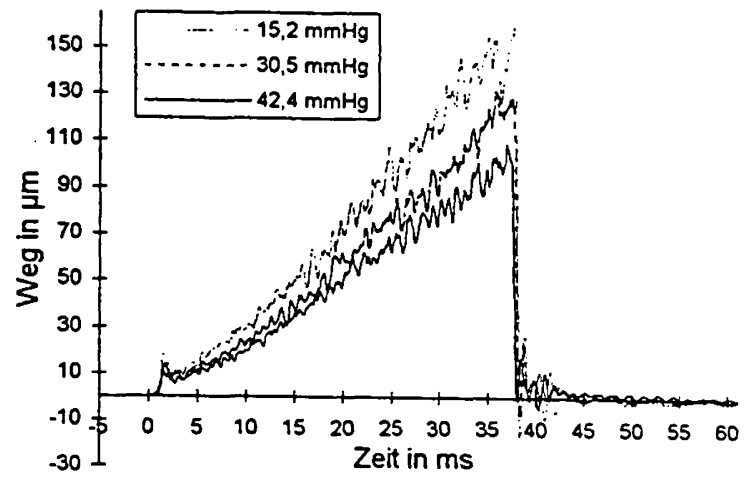

Abb. 2: Verformung von Eichkalotten für Non-ContactTonometer

\section{LITERATUR}

[1] Draeger, J.; Jessen, K.; Rumberger, E.: Tonometrie, Thieme Verlag, Stuttgart 1993.

[2] Büchner, H.; Jäger, G.: Interferometrisches Meßverfahren zur berührungslosen und quasi punktförmigen Antastung von Meßoberflächen. Technisches Messen, 59 (1992), S.43-47.

[3] Lemke, K.; Plagwitz, K.-U.; Rothe. R.: Augenphantom zur Kalibrierung und Überprüfung von Tonometern, Biomed. Technik, 43 (1998), Ergänzungsband 32. Jahrestagung der DGBMT.

Diese Arbeiten wurden vom Thüringer Ministerium für Wissenschaft, Forschung und Kultur gefördert. 